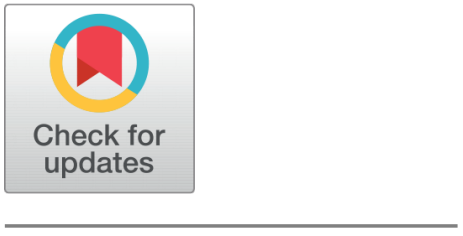

open ACCESS

Received: 13.01.2021

Accepted: 16.05.2021

Published: 03.06 .2021

Citation: Faisal SM, Zaidi T (2021) Implementation of ACO in Vanet with Detection of Faulty Node. Indian Journal of Science and Technology 14(19): 1598-1614. https ://doi.org/10.17485/IJST/v14i19.76

* Corresponding author.

taskeenzaidi867@gmail.com

Funding: None

Competing Interests: None

Copyright: (c) 2021 Faisal \& Zaidi. This is an open access article distributed under the terms of the Creative Commons Attribution License, which permits unrestricted use, distribution, and reproduction in any medium, provided the original author and source are credited.

Published By Indian Society for Education and Environment (iSee)

ISSN

Print: 0974-6846

Electronic: 0974-5645

\section{Implementation of ACO in Vanet with Detection of Faulty Node}

\author{
Syed Mohd Faisal' ${ }^{1}$, Taskeen Zaidi ${ }^{1 *}$ \\ 1 Department of Computer Science and Engineering, Shri Ramswaroop Memorial University, \\ Deva Road, Barabanki, Uttar Pradesh, India
}

\section{Abstract}

Objective: To propose an Ant Colony Optimization (ACO) based routing approach for estimating the reliability parameter of backward and forward ants. Methods: The ACO-based proposed methodology is used to predict path or route discovery in VANET. This study has discovered the best optimal path for data transmission. The forward ant movement has a minimum hop count of four for vehicle number 6 . The vehicle 6 is a source vehicle, and it starts data transmission. The pheromone concentration of the entire vehicle defers and, after updating, the performance was analyzed. The best path is selected based on hop count, then a path with maximum pheromone concentration is selected. The pheromone evaporation factor is updated after a certain time interval. Findings: We have checked the reliability of the path, considering pheromone evaporation as a factor, the pheromone table is updated at a regular time interval, and the reliability parameter is also proposed for performing a task without failure in an efficient manner. We have arranged a routing table in descending order of hop count and then select the best of three paths with a minimum hop count. We search for hop count less than or equal to 5 and total pheromone greater than or equal to 17.55 . We find that Ant 3 having a hop count of 5 and total pheromone concentration of 19.37. In this work, we have taken the evaporation factor as 0.5 . If the transmission of a message is successful, then the reliability assigned to the edge will be 100, and for unsuccessful transmission, reliability is taken down by 20. If the reliability of the path will become 40 , then the vehicle has to search for a new path. In this way, we eliminate malicious nodes for efficient transmission of message. Novelty: The performance of VANET is improved using ACO based proposed routing approach. The optimal path is selected with a minimum hop count and reliability parameter to identify the successful and unsuccessful path.

Keywords: Network security; VANET; Sybil; ACO; Pheromone Evaporation

\section{Introduction}

VANET is a wireless network that is equipped with OBU to connect vehicles with Roadside units. In this study, the forward and backward ant movements were analyzed 
through a modified Ant Colony Optimization algorithm (ACO). The reliability of the path was analyzed to eliminate the broken links or failed links. The movement of ants was modified based on pheromone concentration, and the shortest path was identified by updating the pheromone table and measuring the hop count.

VANET is not a recent technique; during the past two decades, the wireless infrastructure that runs on the Internet has made huge strides and has governed a new wireless world's standards. The VANET is a part of Mobile Ad-hoc Network (MANET) where nodes are smart vehicles ${ }^{(1)}$. VANET aim is to establish and maintain communication between smart vehicles and infrastructure via Dedicated Short Range Communication (DSRC). Foremost, VANET lets vehicles to maintain communication in between vehicles without using any central controller or infrastructure. The essential components of VANET were well explained $^{(2)}$. A modified ACO-based method was proposed ${ }^{(3)}$. A novel cross layer based ACO-AODV was proposed ${ }^{(4)}$. This protocol optimized the information from application layer, transport layer, physical layer and MAC layer in networks. The ACO was used to resolve route selection issue and simulations were performed to evaluate the performance of network. The authors ${ }^{(5)}$ proposed a Flying Ant Colony Optimization(FACO) algorithm for solving the Travelling Salesman Problem(TSP). The new proposed algorithm is dynamic in nature. The results were better than ACO compared through five different methods in terms of quality of solutions. Praveen et al. ${ }^{(6)}$ explain various routing protocols currently available for observing the behavior of ants and it was observed that the modified ACO performs better than existing ACO in terms of cost, propagation delay, packet delivery ratio etc. The authors also reviewed security protocols and enlisted advantages, disadvantages and issues of VANET. The authors Zaidi and Faisal ${ }^{(7)}$ broadly elaborated the working of VANET components and discussed the potential attacks possible over VANET.

\subsection{Ant Colony Optimization}

An improved hybrid ACO method was proposed ${ }^{(8,9)}$ for decreasing the congestion in the network in smart cities. This method is also helpful in improving the quality of service during packet transmission. Efficiency, throughput, packet delivery ratio, end to end delay was simulated using the MATLAB tool. The power-aware optimization approaches in MANETs were discussed. The structures, advantages, design challenges were reviewed and existing power-aware policies were studied to find new research directions ${ }^{(10)}$. An improved Hybrid Ant Particle Optimization(IHAPO) algorithm was proposed to select the best route by removing congested paths during peak hours ${ }^{(11)}$. The IHAPO was proposed using a Graphical Processing Unit(GPU) harness for faster computations. The overall prediction of this algorithm is that it decreases travel time under bulky traffic conditions. Ankita et al. ${ }^{(12)}$ proposed an Adaptive Intersection Selection Mechanism using Ant Colony Optimization (AISM), where authors discover the promising routes to transmit data. Authors first explore a real-time road environment and then break the long path into multiple paths to find the optimum path. The Sybil attack in VANET was discussed by Celestine et al. ${ }^{(13)}$.

A meta-heuristic based Spider-Monkey Technique was proposed that reduces packet loss and propagation delay to maintain the network performance. An Adjusted K Medoids Clustering Algorithm (AKCA) was proposed by authors ${ }^{\text {(14) }}$ for achieving stability in VANET. It was analyzed that clusters created using AKCA have good stability in terms of performance and offers better QOS. Mavrovouniotis et al. ${ }^{(15)}$ reviewed existing swarm intelligence algorithms, and other variations of swam intelligence. The authors also examined Ant colony optimization, bee-inspired algorithms, particle swarm optimization and Fish swarm optimization under different computational problems and suggested some solutions to enhance the performance of the network using Swarm intelligence. The authors Faisal and Zaidi ${ }^{(16)}$ explained the working of VANET and its components. Later, the authors proposed a timestamp-based methodology and VANET network was created for detection of Sybil node using simulation tool. The performance of VANET was estimated through the Fire Fly with Levy Distribution (FF-L) algorithm in the multicast routing problem. The multicasting scenario was analyzed using different experiments. The experimental results determined that FF-L detects optimal routes and can be implemented for network stability ${ }^{(17)}$. An efficient clustering V2V routing based PSO is proposed for VANET ${ }^{(18)}$. The SUMO and NS3 tool was used to analyze the performance of proposed algorithm. It was analyzed that node numbers, node communication radius, hop count influence the performance of VANET. The packet delivery was increased $20 \%$ and delay minimized to $47 \%$ using this approach.

A Quantum based PSO was proposed by authors ${ }^{(19)}$ for End-member Extraction (EE). The experimental results depicts the proposed algorithm performs effectively and efficiently. The PSO approach can be used to search for optimal solutions. Vehicles communicate with other vehicles through Vehicle to Vehicle communication (V2V) and with RSU using Vehicle to Infrastructure communication (V2I) via IEEE 802.11p. If a vehicle in VANET communicates through a hotspot or cellular medium, then IEEE $802.11 \mathrm{a} / \mathrm{b} / \mathrm{g}$ will be used ${ }^{(20)}$ for communication. A novel anomaly detection and characterization algorithms for VANET is proposed.

An improved multi-objective complex network was proposed ${ }^{(21,22)}$ to address the single objective complex network's inefficiency. The PSO search the optimal solution in dynamic spaces. The experimental findings depicts that proposed approach is effective for complex network clustering. A two-fold approach for VANET communication was proposed by authors ${ }^{(23)}$. The 
parameters like received signal strength, transmit power, frequency; path loss was used to design the new metric for VANET. An improved genetic algorithm was proposed for route optimization and better routing. The findings suggested that proposed algorithm improves road communication and reduced road accidents. The performance of VANET was observed through Cross Layer Weighted Position Based Routing (CLWPR) to predict the location of vehicles on GPS ${ }^{(24)}$. The heuristic function for finding the probability of selection of nodes is proposed ${ }^{(25)}$. The formula to evaluate the total pheromone concentration of the path covering next vehicle is explained ${ }^{(26)}$.The real-world ants smell and deposit pheromone wherever they travel, while artificial ants update artificial pheromone during round trip time only ${ }^{(27)}$. An ACO-based algorithm was proposed by combining reactive and proactive scheme. The proposed algorithm is termed as Ant Queue Optimization Scheme (AQO). The performance of proposed scheme is better than the traditional scheme ${ }^{(28)}$. The pheromone concentration updating is evaluated ${ }^{(29)}$. A new Clustering Based Reliable Low Latency Multipath Routing (CRLLR) method was proposed using ACO. The reliability was used as a metric for cluster head selection. The optimal routes were computed, and performance was analyzed on parameters like reliability, E-E delay, throughput and energy consumption. The results demonstrated that reliability is improved with higher energy consumption ${ }^{(30)}$. An adaptive routing protocol based on the Cuckoo search algorithm was proposed which explained the features of topology routing and geographical routing scheme for secure data transmission with less delay. The network performance was slightly improved and latency also decreased ${ }^{(31)}$.

After reviewing the literature it was observed that various research papers proposed ACO based VANET for data transmission, route optimization and congestion removal in VANET but research articles were not discussed in detail about forward ants and backward ants movement for route searching and route optimization in ACO based VANET. The previous work does not explained about the detection of faulty node in network based on reliability of the paths. In the current work if faulty node is detected during route searching it will be removed from pheromone table and new path will be traversed for data transmission based on reliability parameter. The current work is based on selection of the shortest path based on ACO and detection of faulty nodes in VANET. The Bants and Fants were searching the path and sending the packets from source to destination and reversing from destination to source.

The proposed algorithm can be applied to Vehicular ad-hoc Network (VANET) applications like road traffic management, congestion detection in a network, etc. The remaining sections of the article covered potential works on modification of ACO. In the proposed work source node initiates and forward data to destination node and then it checks the entry of destination node in pheromone table and if destination node entry not find in pheromone table then path discovery process will be initiated by source ants. Fants search path between source node and destination node by traversing the network and track the traversed path. The forward ants send data to destination node and increase the hop count. The destination creates backward ants and reverse path is followed; the source node will become destination node and destination will behave as source node. The shortest path is identified based on hop count. The reliability factor analyzed the state of broken links and the selection of alternate paths.

\subsection{Background}

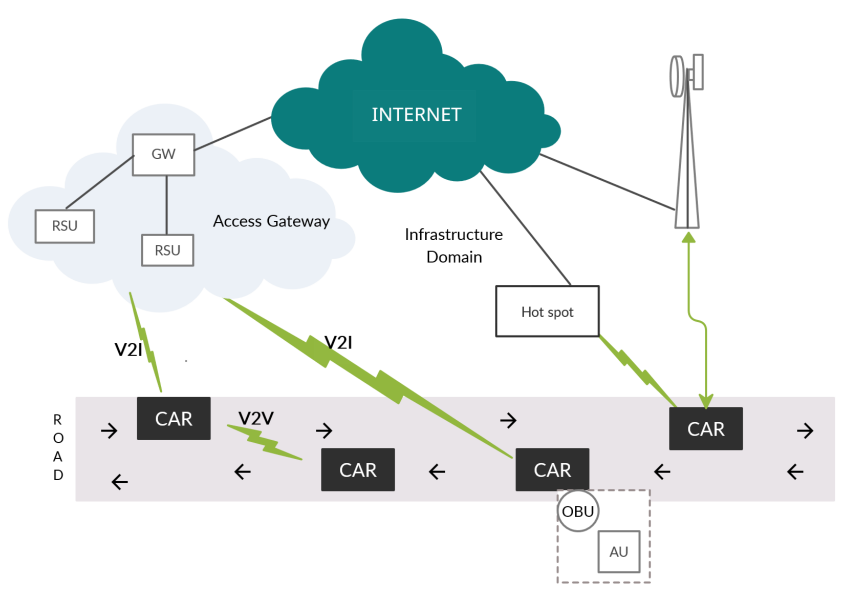

Fig 1. Architecture of VANET 


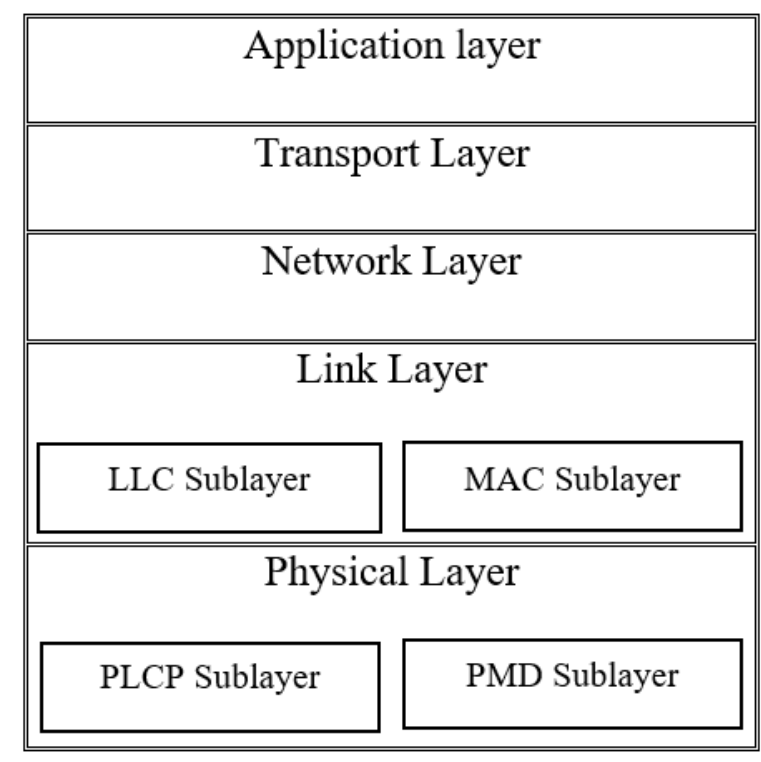

Fig 2. Layered Architecture of VANET

\subsubsection{ACO Based VANET}

The Vehicular Ad-hoc Network (VANET) deploys to provide real-time safety-related information as shown in Figures 1 and 2, ensuring that the driver takes appropriate steps to avoid accidents. When a source node initiates to forward data to a destination, it checks a destination node entry in its pheromone table; if the source node found an entry, it sends data via the next relay node. If the source node does not find the next relay node to the destination in its pheromone table, then the path discovery process is inaugurated by the source node. Their source node forwards a request packet by forward ants (Fants). When a Fant arrives at any node, that node updates its entry in Fants and records the nodes visited by Fants.

In the same way, all intermediate nodes record the data and forward Fants, unless Fants found their destination node or Time to Live (TTL) or Hop Count (HC) will expire. When Fant arrives at the destination node, the destination node acknowledge by creating backward ants (Bants). The destination node forwards Bants to the source node via the reverse route. When backward ants are on the way to acknowledge the source node, they update the pheromone concentration of the path travelled and update the intermediate node database. Table 1 shows the structure of forward and backward ants.

Table 1. Structure of forward and backward ants

\begin{tabular}{llllll}
\hline Source node Address & Destination node Address & Path & Sequence & HC & TTL \\
\hline
\end{tabular}

Backward ants update pheromone concentration, and intermediate nodes update their pheromone table. Pheromone table (Table 2 ) is a two-dimensional array where column $(b, c, d, e, f \ldots)$ denotes neighbor node, row ( $p, q, r, s$...) denotes destination node, and entry in the table is the pheromone concentration on the path. Table 2 is the pheromone concentration table of node "a", where $\Theta b-p$ is pheromone concentration on the link (b, p)

Table 2. Pheromone table for node a

\begin{tabular}{|c|c|c|c|c|c|}
\hline \multirow{2}{*}{ Neighbor } & \multicolumn{5}{|c|}{ Destination } \\
\hline & $\mathrm{p}$ & $\mathrm{q}$ & $\mathrm{r}$ & $\mathrm{s}$ & $\ldots$ \\
\hline $\mathrm{b}$ & $\Theta b-p$ & $\Theta b-q$ & $\Theta b-r$ & $\Theta b-s$ & $\ldots$ \\
\hline c & $\Theta c-p$ & $\Theta c-q$ & $\Theta c-r$ & $\Theta c-s$ & $\ldots$ \\
\hline $\mathrm{d}$ & $\Theta d-p$ & $\Theta d-q$ & $\Theta d-r$ & $\Theta d-s$ & $\ldots$ \\
\hline e & $\Theta e-p$ & $\Theta e-q$ & $\Theta e-r$ & $\Theta e-s$ & $\ldots$ \\
\hline $\mathrm{f}$ & $\Theta f-p$ & $\Theta f-q$ & $\Theta f-r$ & $\Theta f-s$ & $\ldots$ \\
\hline$\ldots \ldots$ & $\ldots$ & $\ldots$ & $\ldots$ & $\ldots$ & $\ldots$ \\
\hline
\end{tabular}




\section{Proposed Methodology}

Route Discovery in VANET through ACO: In this section, we propose a procedure to implement Ant Colony Optimization over VANET. As we know, ants communicate via the stigmergy process and use stochastic process to select the next node to reach the destination node. In this article, we consider VANET network as a graph, where vehicles are nodes of graph and link between vehicles is the path for ants.

It is necessary to understand some distinctions between real-world ants and artificial ants before we start the algorithm:

1. Natural ants exist in the real world and travel asynchronously, while artificial ants live in the discrete world and move synchronously, i.e. from nest to food source and from food source to nest in a finite step through the same path.

2. Real ants perform forging and leaves the same amount of pheromone irrespective of the quantity of food source, while artificial ants update the artificial pheromone based on the quantity and quality of the food source.

\subsection{Forward ants}

\section{Algorithm 01: Ant Colony Optimization Metaheuristic:}

1. Set parameter, initialize pheromone trails.

2. While termination condition do not met, do

a. Construct Ant Based Solution

b. Apply Local Search

c. Update Pheromone

3. end while

Let suppose a source node " $s$ " or vehicle is intended to initiate communication with destination node or vehicle "d". Then source vehicles create a forward ant i.e. Fants and broadcast Fants to all neighbors vehicle of its own. Fants objective is to search path between source node $s$ and destination node $\mathrm{d}$ by traversing the network and track the path traversed. If a vehicle $\mathrm{i}(\mathrm{a}$ vehicle which was last added to the tour) has a path for destination $d$, then vehicle i forward Fants toward next node $j$ (a vehicle which Fants may select) with probability

$$
\begin{gathered}
\text { Probability } i, j=\frac{(\text { Pheromone } \mathrm{i}, \mathrm{j})(\text { Heuristic } \mathrm{i}, \mathrm{j})}{\sum(((\text { Pheromone } \mathrm{i}, \mathrm{j})(\text { Heuristic } \mathrm{i}, \mathrm{j})} \\
P i(j)=\frac{\left(\zeta_{i, j}\right)^{\alpha}\left(\eta_{i, j}\right)^{\beta}}{\sum_{S \varepsilon N_{i}}\left(\left(\zeta_{i, j}\right)^{\alpha}\left(\eta_{i, j}\right)^{\beta}\right)}
\end{gathered}
$$

Where $\mathrm{N}_{i}$ is the set of neighbor nodes of node i, $\alpha$ and $\beta$ are the constant parameters and mostly they assumed to be $1 . \alpha$ is used to regulate the influence of pheromones $(\zeta)$, and $\beta$ is used to regulate the influence of heuristic information $(\eta) . \eta$ is also known as the weight function for calculating the hop count in VANET, so we consider no weight (zero) on the edges. After considering the heuristic function as zero, then the probability of selection of nodes is proposed.

$$
P i(j)=\frac{\left(\zeta_{i, j}\right)^{\alpha}}{\sum_{S \varepsilon N_{i}}\left(\left(\zeta_{i, j}\right)^{\alpha}\right)}
$$

Algorithm 02: Forward Ant (Fants) (sender is "s" and destination is "d")

1. If sender vehicle "s" has an entry of destination "d" in its pheromone table: start data transmission. Else: go to step 2.

2. Sender vehicle "s" broadcast Fants

3. Intermediate vehicle ("k") checks its pheromone table for "d".

(a) If destination vehicle, "d" found go to step 4, Else

(b) Increase Hop Count

(c) Select next vehicle $\mathrm{j}$ with probability $\mathrm{Pi}(\mathrm{j})$ and forward Fants.

4. If destination "d" arrived, Go to Algorithm 03, Else go to step 03 


\section{Exit}

After exploring multiple paths, Fants reached the destination vehicle "d" forthwith destination vehicle creates backward ants (Bants). The function of Bants is to take the same route followed by Fants and reach the source vehicle. When Bants are on the way to reach the source vehicle, they update pheromone of the path they traverse and mark updated pheromone on the intermediate vehicles. In the same way, all Bants following multiple routes reach the destination ("s") where node "s" calculates an optimum path to reach the node ("d") and start data transmission via calculated optimum path.

\subsection{Backward Ants}

Destination d, create backward ants or Bants. Destination vehicle reverses the source address and destination address, i.e. now the source vehicle is $d$ and destination vehicle is $s$. The function of Bants is to take the same route followed by Fants and reach to the destination vehicle. After the acknowledgement destination vehicle generates Bants, which follows the same route as followed by Fants and reach the destination vehicle (s), in between backward ants update pheromone table of intermediate vehicle and checks the reliability of the edge, if reliability is greater than 40 then that edge is added to the tour of Bants otherwise (if reliability is less than 40) intermediate vehicle search new path between vehicles and inform destination vehicle (s) about new path. Before adding a vehicle to the tour, Bants checks pheromone concentration on path i.e. $\varphi_{i, j}$, if reliability check is successful then vehicle calculates and update pheromone concentration $\zeta_{i, j}$ of path i-j in pheromone table of vehicle i.

$$
\zeta_{i, j}=\varphi_{i, j}+\frac{1}{H C}
$$

As a new edge is added to the tour so Bants update total pheromone concentration $\triangle \zeta_{i, j}$ of the path and then proceed towards next vehicle.

$$
\triangle \zeta_{i, j}=\triangle \zeta_{i, j}+\zeta_{i, j}
$$

Algorithm 03: Backward Ants (Bants) (The Sender is "d", destination is "s", " $\varphi_{i, j}$ " is the pheromone concentration of next edge to be select, updated pheromone concentration on path $i-j$ is " $\zeta_{i, j}$ ", Hop Count is HC)

1. Node d generates Bants and check for the destination node (s).

2. If vehicle $==$ destination vehicle:

(a) Update Hop (HC) by $\mathrm{HC}=\mathrm{HC}+1$

(b) Update pheromone table of destination vehicle $\left(\mathrm{V}_{s}\right)$ by, $\zeta_{s, j}$.

(c) Update routing table of vehicle "s" by total pheromone concentration on path "s-d" search by ant k. Else go to step 3

3. Update hop-count ( $\mathrm{HC}$ ) by $\mathrm{HC}=\mathrm{HC}+1$

4. If vehicle $==$ source vehicle: Set $\Delta \zeta_{i, j}=0, \zeta_{i, j}=0$ and $\mathrm{HC}=1$.

Else Update pheromone table of vehicle $\left(\mathrm{V}_{i}\right)$ by $\zeta_{i, j}$.

5. Check next edge pheromone in the pheromone table for $\varphi_{i, j}$. Go to Algorithm 04

6. Update Pheromone table of vehicle $\mathrm{V}_{i}$, $\zeta_{i, j}=\varphi_{i, j}+\frac{1}{H C}$

7. Update Total Pheromone of path, $\Delta \zeta_{i, j}=\Delta \zeta_{i, j}+\zeta_{i, j}$

8. Reach to the next vehicle, go to step 2 . 


\subsection{Path Lost}

Meanwhile, due to the congestion or swift nature of the vehicles in VANET we can't ignore the link failure between vehicles. For checking the effective path we define reliability parameter. The purpose of reliability parameter is to find correct path between vehicles, and for this, we checked the reliability of the edge; if the reliability of edge is less than our numeric constant, we believe that edge is not fit otherwise, edge is fit. Whenever an intermediate vehicle detects any unfit edge it forwards a new-Fants (execute algorithm 2) to search for the destination node; If an intermediate vehicle receives new-Bants it updates its pheromone table using the pheromone concentration of new-Bants otherwise it sends a "Path Lost" message to the source vehicle.

Algorithm 0 4: Check the reliability of the path

1. If Reliability (R) of Edge $\varphi_{i, j}$ is greater than 40 (i.e. $\mathrm{RE}_{i, j}>=40$ ), Goto Algorithm 03, Step 6.

Else: Path is not reliable, search alternate path (Step 2).

2. Vehicle $\left(\mathrm{V}_{m}\right)$ broadcast a new-Fants and search for destination between $\mathrm{V}_{m}-\mathrm{V}_{n}$.

(a) If path found:

i. Vehicle $\mathrm{V}_{m}, \mathrm{~V}_{n}$ update new-path and Pheromone table.

ii. Vehicle $\mathrm{V}_{m}$ forward message to vehicle $\mathrm{V}_{s}$ about the new path between $\mathrm{V}_{m}$ and $\mathrm{V}_{n}$.

(b) Else

i. Vehicle $\left(\mathrm{V}_{m}\right)$ remove its entry of $\mathrm{V}_{m}-\mathrm{V}_{n}$ in its Pheromone table.

ii. Transmit a "Path Lose" message to Vehicle $\mathrm{V}_{s}$

\subsection{Pheromone Evaporation and Reliability Calculation}

Most of the authors have taken random assumption on pheromone evaporation, various researchers and scholars proposed different methods to update pheromone, some uniformly update the pheromone and other update the pheromone based on the optimal solution. A large class of scholars proposed pheromone trail update after calculating the optimal solution but in this article we update the pheromone trail at every vehicle/node and uniformly assuming that the pheromone concentration decreases on all edges. To implement this approach, we update pheromone on vehicles, i.e., after $n$ interval of time, all vehicles are required to update the pheromone concentration of their directly connected edge.

\subsection{Pheromone Initialization}

In the initial construction process, each path has an equal number of pheromone concentrations, which implies that if a search process initiates, then every node broadcast Fants to search for a destination.

$$
\zeta_{i, j}=\frac{1}{\mid \text { Vehicles } \mid}
$$

Here |Vehicles| is number of vehicles in network and $\zeta_{i, j}$ is pheromone concentration on path $\mathrm{i}, \mathrm{j}$.

We can initialize the pheromone based on the density of vehicles in network, the number of vehicles towards a specific path is dense, and we can initialize high pheromone concentration toward that path/region or vice versa.

\subsection{Pheromone Update}

The Intention behind pheromone update is to increase the pheromone concentration for the good path and decrease the pheromone concentration towards bad path. This concept is achieved by increasing pheromone towards optimal solution and decrease pheromone concentration for unfit/faulty path (Pheromone Evaporation).

A different ACO variant uses different pheromone update parameters. One method is to update pheromone every time an ant traverses between two nodes, updating the pheromone level by 1 between the vehicles only. Another method is to update the pheromone value based on the length of the tour.

In this paper, we use the basic ACO pheromone update procedure. In ACO each ant constructs its own path so the concentration of pheromone between vehicles $\mathrm{i}$ and $\mathrm{j}$ by $\mathrm{k}_{t h}$ is [15].

$$
\Delta \zeta_{\mathbf{i}, \mathbf{j}}^{\mathbf{k}}= \begin{cases}\mathbf{Q} / \mathbf{L}_{\mathbf{k}} & \mathbf{k}_{\mathrm{th}} \text { ant traverse on edge } \mathbf{i}, \mathbf{j} \\ 0 & \text { otherwise }\end{cases}
$$

https://www.indjst.org/ 
Where $i, j$ shows the edge connecting vehicle $i$ and vehicle $j, k$ is the ant which is traversing between node $i$ and $j$. The positive constant Q is the parameter of the mode (mostly Q is 1 ), $\mathrm{L}_{k}$ is the length of the path found by $\mathrm{k}_{t h}$ ant, $\triangle \zeta$ is the amount of pheromone deposited by an ant. $\triangle \zeta_{i, j}^{k}$ is the total amount of pheromone deposited by ant $\mathrm{k}$ between path $\mathrm{i}$ and $\mathrm{j}$.

If ants traverse on a route then the pheromone is $\frac{Q}{L_{k}}$ i.e. $\frac{1}{L_{k}}$ otherwise 0 : if ant $\mathrm{k}$ does not choose any edge/path for tour. We can say that the amount of pheromone is inversely proportional to the length of the path, i.e., the shortest path has a high amount of pheromone concentration, and longer path have a low amount of pheromone concentration.

Similarly, multiple ant follows same route so the amount of pheromone deposited by multiple ants is:

$$
\zeta_{i, j}^{k}=\sum_{k=1}^{m} \triangle \zeta_{i, j}^{k}
$$

Where $\mathrm{m}$ is the total number of ants, $\triangle \zeta_{i, j}^{k}$ is the quantity of pheromone deposited by ant $\mathrm{k}$. The above equation shows the amount of pheromone between $i$ and $j$, when there is no evaporation. To find the best path it is essential to consider pheromone evaporation. So the rate of pheromone evaporation is:

$$
\zeta_{i, j}=(1-\rho) \zeta_{i, j}
$$

Where $\rho$ is the evaporation rate, i.e. if value of $\rho$ is 0 , there is no evaporation and if $\rho$ is 1 that means evaporation is at its climax. $\zeta_{i, j}$ is the current pheromone level.

After taking evaporation rate in consideration the amount of pheromone deposited on edge $i$ and $j$ is:

$$
\zeta_{i, j}^{k}=(1-\rho) \zeta_{i, j}+\sum_{k=1}^{m} \triangle \zeta_{i, j}^{k}
$$

where $\sum_{k=1}^{m} \triangle \zeta_{i, j}^{k}$ is the new pheromone deposited by ants.

So, this is the pheromone update parameters in Ant colony optimization. In the next section we will study how ACO metaheuristic works on VANET.

\section{Results and Discussion}

Initially, we assume that: i) Artificial arts have memory (T) so, they will not visit the vehicle which they have already visited. ii) Not all ants are allowed to update the pheromone table; only backward ants (Bants) are accredited to update the pheromone table.

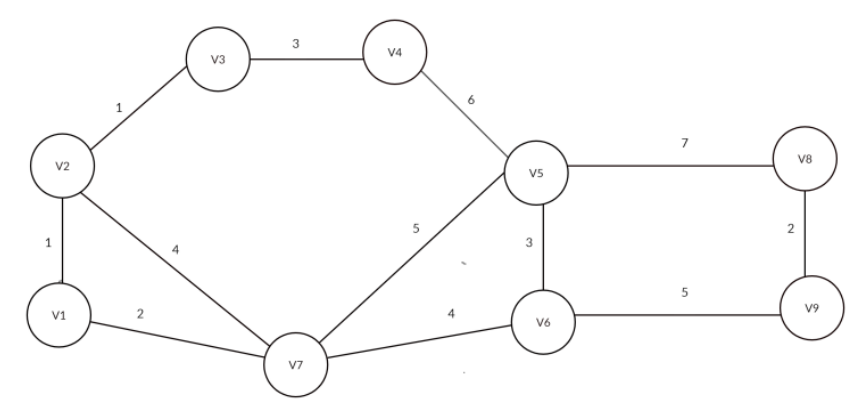

Fig 3. Graph of Network

Initially, we consider vehicle $\mathrm{i}$ forwards $\mathrm{k}$ ants to search for destinations, considering that network is congestion-free and $\mathrm{k}$ number of backwards ants arrives at the vehicle $i$, after route discovery. Now the source node starts creating routing table as soon as backward ants start arriving at same. Below is the layout of the pheromone table stored at each node: where $\mathrm{x}$ and $\mathrm{y}$ is the intermediate nodes and 2 are the pheromone concentration on path $x-y$.

And if $\mathrm{k}$ numbers of backward ants have not arrived, we follow different mechanisms to find the optimal path.

At the beginning source vehicle sends some (let say " $k$ ") forward ants; forward ants' checks for the destination in its pheromone table if destination; found, then ants visit the destination, otherwise forward ants search for destination (see 
Table 3. Pheromone Table

\begin{tabular}{lll}
\hline S.No & Route & Total Pheromone on that route \\
\hline 1 & $\mathrm{x}-\mathrm{y}$ & 2 \\
2 & $\mathrm{~h}-\mathrm{z}$ & 6 \\
$\ldots$ & $\ldots \ldots \ldots \ldots$ & $\ldots \ldots \ldots \ldots \ldots$ \\
\hline
\end{tabular}

algorithm 2). After exploring multiple paths when forward ants arrived at destination, the destination vehicle checks the packet's validity and creates backward ants. The task of backward ants is to update the pheromone table of the path followed by forward ants, update the hop count and calculate the total pheromone concentration on that path. Consider figure 07 is the graph of the network where vehicle V1 is the source vehicle and vehicle V9 is destination vehicle. Vehicle V1 sends multiple forward ants to search for destination V9, and after investigating multiple paths, forward ants reach the destination.

Table 4. Path discovered by ants

\begin{tabular}{ll}
\hline Ant & Path Found \\
\hline 1 & $\mathrm{~V} 1-\mathrm{V} 7-\mathrm{V} 6-\mathrm{V} 9$ \\
2 & $\mathrm{~V} 1-\mathrm{V} 2-\mathrm{V} 7-\mathrm{V} 6-\mathrm{V} 9$ \\
3 & $\mathrm{~V} 1-\mathrm{V} 7-\mathrm{V} 5-\mathrm{V} 6-\mathrm{V} 9$ \\
4 & $\mathrm{~V} 1-\mathrm{V} 7-\mathrm{V} 5-\mathrm{V} 8-\mathrm{V} 9$ \\
5 & $\mathrm{~V} 1-\mathrm{V} 2-\mathrm{V} 7-\mathrm{V} 5-\mathrm{V} 8-\mathrm{V} 9$ \\
6 & $\mathrm{~V} 1-\mathrm{V} 2-\mathrm{V} 7-\mathrm{V} 5-\mathrm{V} 6-\mathrm{V} 9$ \\
7 & $\mathrm{~V} 1-\mathrm{V} 2-\mathrm{V} 3-\mathrm{V} 4-\mathrm{V} 5-\mathrm{V} 8-\mathrm{V} 9$ \\
8 & $\mathrm{~V} 1-\mathrm{V} 2-\mathrm{V} 3-\mathrm{V} 4-\mathrm{V} 5-\mathrm{V} 6-\mathrm{V} 9$ \\
\hline
\end{tabular}

When forward ants reached at V9 thereupon V9 creates backward ants(Bants) and forward Bants to destination (for backward ants, source vehicle is $\mathrm{V} 9$ and destination vehicle is V1).

Consider that ant 1 (follow path V1 - V7 -V6 -V9) reach first at destination then backward ant 1 follow path V9 - V6 - V7 - V1 and reach to vehicle V1. Initially, backward ant checks whether this node is destination node or not if this node is not a destination node, then backward ants first update hop count $(\mathrm{HC}=\mathrm{HC}+1)$, next it checks whether this node is source node or not. For the first time when backward ants is on vehicle V9 then backward ants are initialized with total pheromone $\left(\triangle \zeta_{i, j}\right)=$ pheromone update in routing table $\left(\triangle \zeta_{i, j}\right)=0$ and Hop Count $(\mathrm{HC})=1$. Thenceforth we check the pheromone concentration on next edge $(\varphi)$ (i.e. V9-V6) which is $5\left(\varphi_{9,6}=5\right)$ so backward ant update pheromone table of vehicle $\mathrm{V} 9$ by $\zeta_{i, j}=\varphi_{i, j}+\frac{1}{H C}$, $\left(\zeta_{9,6}=5+\frac{1}{1}=6\right)$ and update total pheromone concentration on the edge of V9 and V6 by $\triangle \zeta_{9,6}=6$.

Table 5. Pheromone table before update

\begin{tabular}{ll}
\hline \multicolumn{2}{l}{ Pheromone table of Vehicle V9 } \\
\hline Next node & Pheromone Concentration \\
\hline 6 & 5 \\
8 & 2 \\
\hline
\end{tabular}

Table 6. Pheromone table after update

\begin{tabular}{ll}
\hline \multicolumn{2}{l}{ Pheromone table of Vehicle V9 } \\
\hline Next node & Pheromone Concentration \\
\hline 6 & 6 \\
8 & 2 \\
\hline
\end{tabular}

Later backward ants reach at vehicle V6, here first we update hop count $(\mathrm{HC}=2)$ and update a pheromone table of vehicle V6 by $\varphi_{9,6}=6$ (See Tables 7 and 8). The next phase is to check source node and destination node; here vehicle V6 is neither a source vehicle nor a destination vehicle so ants will shift to next step. Next step is to check the pheromone concentration on the next edge i.e. V6 - V7 in the pheromone table of V6 which is $\varphi_{6,7}=4$. Now we update pheromone table of the vehicle 
V6 by $\zeta_{i, j}=\varphi_{i, j}+\frac{1}{H C}$ (see Tables 9 and 10) $\left(\zeta_{6,7}=4+\frac{1}{2}=4.5\right)$ and update total pheromone concentration on the path by $\triangle \zeta_{i, j}=\triangle \zeta_{i, j}+\zeta_{i, j}$. So total pheromone is $\triangle \zeta_{9,7}=6+4.5=10.5$.

Table 7. Pheromone table before update

\begin{tabular}{ll}
\hline \multicolumn{2}{l}{ Pheromone table of Vehicle V6 } \\
\hline Next node & Pheromone Concentration \\
\hline 5 & 3 \\
7 & 4 \\
9 & 5 \\
\hline
\end{tabular}

Table 8. Pheromone table after update

\begin{tabular}{ll}
\hline \multicolumn{2}{l}{ Pheromone table of Vehicle V6 } \\
\hline Next node & Pheromone Concentration \\
\hline 5 & 3 \\
7 & 4 \\
9 & 6 \\
\hline
\end{tabular}

Table 9. Pheromone table before update

\begin{tabular}{ll}
\hline \multicolumn{2}{l}{ Pheromone table of Vehicle V6 } \\
\hline Next node & Pheromone Concentration \\
\hline 5 & 3 \\
7 & 4 \\
9 & 6 \\
\hline
\end{tabular}

Table 10. Pheromone table after update

\begin{tabular}{ll}
\hline \multicolumn{2}{l}{ Pheromone table of Vehicle V6 } \\
\hline Next node & Pheromone Concentration \\
\hline 5 & 3 \\
7 & 4.5 \\
9 & 6 \\
\hline
\end{tabular}

After updating the pheromone table of Vehicle V6 and total pheromones, now ants move towards vehicle V7. By analyzing, ants know that vehicle V7 is neither a source vehicle nor a destination vehicle, so they update Hop Count (HC=3) and pheromone concentration of vehicle V7 by $\varphi_{6,7}=4.5$ (See Tables 11 and 12 ).

Table 11. Pheromone table before update

\begin{tabular}{ll}
\hline \multicolumn{2}{l}{ Pheromone table of Vehicle V7 } \\
\hline Next node & Pheromone Concentration \\
\hline 1 & 2 \\
2 & 4 \\
5 & 5 \\
6 & 4 \\
\hline
\end{tabular}

The next step is to check the pheromone concentration on next path, i.e. between V7 and V1. Here pheromone concentration $\varphi_{7,1}=2$, so we update the pheromone table of Vehicle V7 by $\zeta_{i, j}=\varphi_{i, j}+\frac{1}{H C}$ and then calculate the total pheromone concentration on path. $\triangle \zeta_{i, j}=\Delta \zeta_{i, j}+\zeta_{i, j}$

$$
\triangle \zeta_{9,1}=10.5+2.33=12.83
$$


Table 12. Pheromone table after update

\begin{tabular}{ll}
\hline \multicolumn{2}{l}{ Pheromone table of Vehicle V7 } \\
\hline Next node & Pheromone Concentration \\
\hline 1 & 2 \\
2 & 4 \\
5 & 5 \\
6 & 4.5 \\
\hline
\end{tabular}

Table 13. Pheromone table before update

\begin{tabular}{ll}
\hline \multicolumn{2}{l}{ Pheromone table of Vehicle V7 } \\
\hline Next node & Pheromone Concentration \\
\hline 1 & 2 \\
2 & 4 \\
5 & 5 \\
6 & 4.5 \\
\hline
\end{tabular}

Table 14. Pheromone table afterupdate

\begin{tabular}{ll}
\hline \multicolumn{2}{l}{ Pheromone table of Vehicle V7 } \\
\hline Next node & Pheromone Concentration \\
\hline 1 & 2.33 \\
2 & 4 \\
5 & 5 \\
6 & 4.5 \\
\hline
\end{tabular}

The ant finally reaches at vehicle V1, at V1 ants checks the for destination node and found that this node is a destination node. Before performing any task ant first updates Hop Count $(\mathrm{HC}=4)$ and pheromone table of vehicle V1 by $\zeta_{7,1}=2.33$ (See Tables 16 and 17) and later calculate total pheromone concentration. So at the end of first ant traversal following information is constituted (Table 15):

Table 15. Discovered path by ant 1

\begin{tabular}{ll}
\hline \multicolumn{2}{l}{ Information found from ant 1} \\
\hline Source & V1 \\
\hline Destination & V9 \\
Path & V1-V7 -V6-V9 \\
Hop Count & 4 \\
Total Pheromone & 12.83 \\
\hline
\end{tabular}

Table 16. Pheromone table before update

\begin{tabular}{ll}
\hline \multicolumn{2}{l}{ Pheromone table of Vehicle V1 } \\
\hline Next node & Pheromone Concentration \\
\hline 2 & 1 \\
7 & 2 \\
\hline
\end{tabular}

Similarly, multiple ants follow multiple paths and reached at Vehicle V1. This process continues until the number of ants forwarded by vehicle V1 is not equal to number of backward ants arrived at vehicle V1 or route discovery time will expire. After $\mathrm{t}$ time interval vehicle V1 have multiple paths between V1 and V9, they are:

We have already discussed how backward ants update pheromone table of the vehicle, so by the time all backward ants arrived at vehicle V1 or route discovery time will expire; all intermediate nodes have updated the pheromone table.

In our proposed work we are not only finding the best path, but we also find the optimal path for data transmission. Before selecting a path for the data transmission, the source vehicle(VI) may have two possible cases: 
Table 17. Pheromone table after update

\begin{tabular}{ll}
\hline \multicolumn{2}{l}{ Pheromone table of Vehicle V1 } \\
\hline Next node & Pheromone Concentration \\
\hline 2 & 1 \\
7 & 2.33 \\
\hline
\end{tabular}

Table 18. Paths found by multiple backward ants

\begin{tabular}{llllll}
\hline Ant & Source vehicle & Destination vehicle & Path & Hop Count & Total Pheromone \\
\hline 1. & V1 & V9 & V1-V7-V6-V9 & 4 & 12.83 \\
2. & V1 & V9 & V1-V2-V7-V6-V9 & 5 & 17.55 \\
3. & V1 & V9 & V1-V7-V5-V6-V9 & 5 & 19.37 \\
4. & V1 & V9 & V1-V7-V5-V8-V9 & 5 & 18.92 \\
5. & V1 & V9 & V1-V2-V7-V5-V6-V9 & 6 & 23.90 \\
6. & V1 & V9 & V1-V2-V3-V4-V5-V8-V9 & 7 & 25.65 \\
7. & V1 & V9 & V1-V2-V3-V4-V5-V6-V9 & 7 & 26.06 \\
8. & V1 & V9 & & 27.97 \\
\hline
\end{tabular}

Table 19. Pheromone table of Vehicle V1

\begin{tabular}{ll}
\hline \multicolumn{2}{l}{ Pheromone table of Vehicle V1 } \\
\hline Next node & Pheromone Concentration \\
\hline 2 & 1.97 \\
7 & 2.82 \\
\hline
\end{tabular}

Table 20. Pheromone table of Vehicle V2

\begin{tabular}{ll}
\hline \multicolumn{2}{l}{ Pheromone table of Vehicle V2 } \\
\hline Next node & Pheromone Concentration \\
\hline 1 & 1.97 \\
3 & 1.4 \\
7 & 4.8 \\
\hline
\end{tabular}

Table 21. Pheromone table of Vehicle V3

\begin{tabular}{ll}
\hline \multicolumn{2}{l}{ Pheromone table of Vehicle V3 } \\
\hline Next node & Pheromone Concentration \\
\hline 2 & 1.4 \\
4 & 3.5
\end{tabular}

Table 22. Pheromone table of Vehicle V4

\begin{tabular}{ll}
\hline \multicolumn{2}{l}{ Pheromone table of Vehicle V4 } \\
\hline Next node & Pheromone Concentration \\
\hline 3 & 3.5 \\
5 & 6.6 \\
\hline
\end{tabular}

Table 23. Pheromone table of Vehicle V5

\begin{tabular}{ll}
\hline \multicolumn{2}{l}{ Pheromone table of Vehicle V5 } \\
\hline Next node & Pheromone Concentration \\
\hline 4 & 6.6 \\
6 & 4.5 \\
7 & 6.2 \\
8 & 8.5 \\
\hline
\end{tabular}


Table 24. Pheromone table of Vehicle V6

\begin{tabular}{ll}
\hline \multicolumn{2}{l}{ Pheromone table of Vehicle V6 } \\
\hline Next node & Pheromone Concentration \\
\hline 5 & 4.5 \\
7 & 5 \\
9 & 10 \\
\hline
\end{tabular}

Table 25. Pheromone table of Vehicle V7

\begin{tabular}{ll}
\hline \multicolumn{2}{l}{ Pheromone table of Vehicle V7 } \\
\hline Next node & Pheromone Concentration \\
\hline 1 & 2.82 \\
2 & 4.8 \\
5 & 6.2 \\
6 & 5 \\
\hline
\end{tabular}

Table 26. Pheromone table of Vehicle V8

\begin{tabular}{ll}
\hline \multicolumn{2}{l}{ Pheromone table of Vehicle V8 } \\
\hline Next node & Pheromone Concentration \\
\hline 5 & 8.5 \\
9 & 5 \\
\hline
\end{tabular}

Table 27. Pheromone table of Vehicle V9

\begin{tabular}{ll}
\hline \multicolumn{2}{l}{ Pheromone table of Vehicle V9 } \\
\hline Next node & Pheromone Concentration \\
\hline 6 & 10 \\
8 & 5 \\
\hline
\end{tabular}

\section{CASE 01}

Number of forward ants transmitted $==$ Number of backward ants arrived:

In this case the vehicle will select the best path for the data transmission. The best path is selected on the basis of hop count; if multiple paths have a same hop count, then path with maximum total pheromone concentration is selected (lower the hop count, higher the selection chances).

In our example; path V1-V7-V6-V9 having minimum hop count 04, so vehicle V1 will select this path and start data transmission.

\section{CASE 02}

Number of forward ants transmitted! = Number of backward ants arrived:

It is not mandatory that all backward ants arrived at vehicle V1; there were several paths, and it is not possible that all forwarding and backward ants reached at their destination. Some ants may lose destination or some ants may face link failure, taking these assumptions in knowledge we design a method to find the optimum path between source and destination.

Consider the same graph (Figure 07), where V1 is the source node and V9 is destination node. Suppose some backward ants have not arrived at vehicle V1. In that case we will select the optimal path since it is not necessary that path having the least $\mathrm{HC}$ was congestion-free and have maximum pheromone concentration and vice versa. Another important parameter which that we need to notice is that if ants take long route to reach the destination and revert back, then definitely total pheromone concentration of that ant will increase. After analyzing these issues we develop a method to find the optimal path.

1. Arrange tables in ascending order of HC (Table 28).

2. Select $\mathrm{n}$ ants tour with ascending order of HC (Table 29 ).

3. Calculate $\mathrm{PH}=$ Pheromone concentration $\left(\mathrm{Ph}_{i}\right) *$ Hop Count $\left(\mathrm{HC}_{i}\right)$.

4. Select Pheromone concentration $\left(\mathrm{Ph}_{j}\right)$ and $\mathrm{HC}\left(\mathrm{HC}_{j}\right)$ from minimum of $\mathrm{PH}_{i \ldots . . z}$.

5. Compare with Table 28 
(a) If $H C \leq P H\left(H C_{j}\right)$ and $P h \geq P H\left(P h_{j}\right)$

Select this tour and transfer data

Else.

(b) select tour from Table 29 having minimum $\mathrm{PH}$ and start data transmission.

Assuming that due to congestion only limited ants reach at vehicle V1, so vehicle V1 need to select the best path for data transmission, where number of nodes are less and number of pheromone concentration is high. For the accomplishment of this task we apply some numerical technique on routing table.

First of all routing table will be arranged in ascending order of hop count. We then select best of 3 paths with minimum hop count and calculate multiplication of hop count and pheromone concentration (Table 29).

Later we select the least value from PH: in our case it is 87.75, which is ant 2 where hop count is 5 and total pheromone is 17.55 (Table 29 ).

At this moment we search hop count less than or equal to 5 and total pheromone greater than or equal to 17.55 in table 29 . We found that ant 3 having hop count 5 and total pheromone 19.37 is satisfying our condition (Table 28 ). So path found by ant 3 is selected and transmission of data started via this path (V1-V7-V5-V6-V9).

Table 28. Path found by multiple backward ants (Not all)

\begin{tabular}{llllll}
\hline Ant & Source vehicle & Destination vehicle & Path & Hop Count (HC) & Total Pheromone (Ph) \\
\hline 2 & V1 & V9 & V1-V2-V7-V6-V9 & 5 & 17.55 \\
3 & V1 & V9 & V1-V7-V5-V6-V9 & 5 & 19.37 \\
5 & V1 & V9 & V1-V2-V7-V5-V8-V9 & 6 & 23.90 \\
6 & V1 & V9 & V1-V2-V3-V4-V5-V6-V9 & 7 & 25.65 \\
8 & V1 & V9 & 7 & 27.97 \\
\hline
\end{tabular}

Table 29. Path found by multiple backward ants (Not all)

\begin{tabular}{lllllll}
\hline Ant & Source vehicle & Destination vehicle & Path & Hop Count $(\mathrm{HC})$ & $\begin{array}{l}\text { Total } \\
(\mathrm{Ph})\end{array}$ & Pheromone \\
& & & & $\mathrm{PH}=\mathrm{HC} \mathrm{Ph}^{*}$ \\
\hline 2 & V1 & V9 & V1-V2-V7-V6-V9 & 5 & 17.55 & 87.75 \\
3 & V1 & V9 & V1-V7-V5-V6-V9 & 5 & 19.37 & 96.85 \\
5 & V1 & V9 & V1-V2-V7-V5-V8- & 6 & 23.90 & 143.40 \\
& & V9 & & & \\
\hline
\end{tabular}

\subsection{Pheromone Evaporation}

Different researchers proposed different criteria of pheromone evaporation

Ants lay down the pheromone on the path they travel and after some constant time those pheromone start evaporating. It is irremissible to oversee evaporation factor, as if the evaporation factor is too high, then all pheromone get dissipate and on the off chance if evaporation factor is too low then pheromone on that path become static.

$\rho$ is a pheromone evaporation factor and $\zeta_{i, j}$ is the pheromone between vehicle $\mathrm{i}$ and $\mathrm{j}$.

Considering the pheromone evaporation as a vital factor in ACO, we update the pheromone table of every vehicle and pheromone evaporation after a fix time interval (refer pheromone evaporation factor). Here $\rho$ is a pheromone evaporation factor between 0 and 1 but regulation of evaporation factor totally depends on the researcher, in our work we consider the evaporation factor as 0.5 .

\section{Pheromone evaporation factor}

After every n interval vehicle perform these operations:

Update pheromone table

$$
\zeta_{i, j}=(1-\rho) \zeta_{i, j}
$$

\subsection{Reliability Parameter}

We propose another important parameter which is reliability. Reliability is defined as a parameter of performing a task in an environment without failure. We implement reliability parameter to remove unused/irrational edges. We consider that initially reliability of every path is 100 , as soon as transmission starts by that time reliability of the path start varying. If a transmission 
is successful, then the reliability of that edge is assigned with 100 and if the transmission via that path is unsuccessful then we degrade the reliability by $\xi$. In our work we consider $\xi$ as 20 , which means after every unsuccessful transmission reliability of path is decreased by 20 and if reliability of path is less than or equal to 40 we will not follow that path. As soon as reliability reaches at 40 vehicles are bound to authenticate that path or search an alternate path. By this method we eliminate broken path and found new and used path for the efficient transmission.

\section{Reliability Parameter}

1. If transmission done: Reliability $_{i, j}=100$

2. Else: Reliability $_{i, j}=100-\xi$

\subsection{Flowchart of Proposed Algorithm}

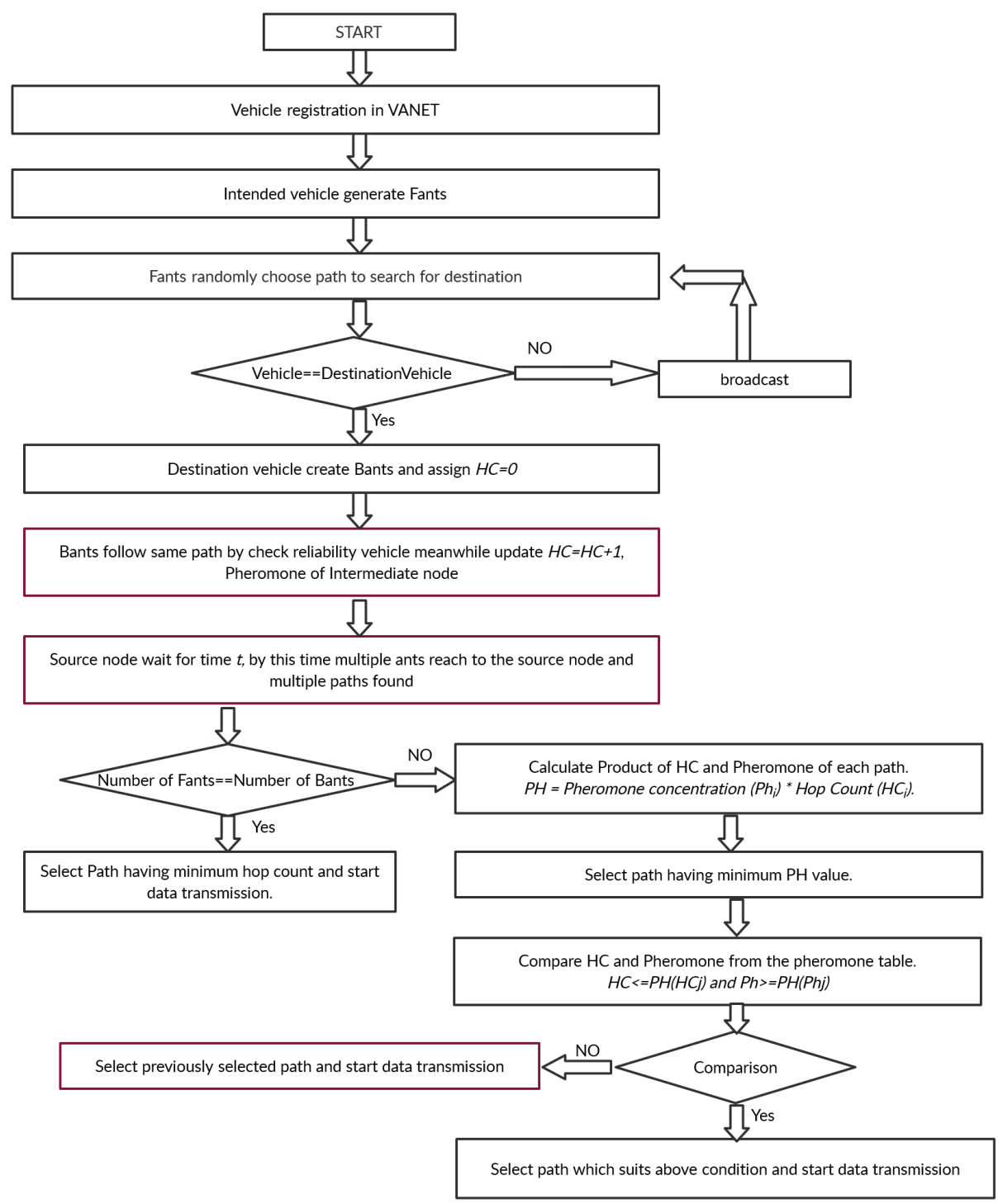

Fig 4. Flowchart of Proposed Algorithm 


\section{Conclusion}

In this work an optimization technique ACO is used for route discovery and reliability parameter is used to provide efficient data transmission by detecting new path and eliminating broken link in the network. In the future we will propose a technique to identify Sybil nodes in VANET using the ACO. In the given work the authors discovered path from source to destination using Ant Colony Optimization approach. In this approach forward ants check a pheromone table to find destination node. After exploring multiple paths the forward ants reached the destination node, then checked for the validity of the packet and creates backward ants. The backward ants, update the pheromone table using the path followed by forward ants, the hop count is updated and total pheromone concentration is calculated. The reliability parameter checks for valid and invalid edges. It was analyzed that if the reliability of path is less than or equal to 40 , the nodes will discard that path and start searching for an alternate path for data transmission. In the past authors have proposed a modified ACO with DBA for computation of the distance between the vehicles but reliability was not analyzed. In future, we will extend the work of discovering the distance between the vehicles using evolutionary approach.

\section{References}

1) Ghori MR, Zamli KZ, Quosthoni N, Hisyam M, Montaser M. Vehicular ad-hoc network (VANET): Review. In: IEEE International Conference on Innovative Research and Development (ICIRD). 2018;p. 1-6. Available from: https://doi.org/10.1109/ICIRD.2018.8376311.

2) Hu S, Jia Y, She C. Performance Analysis of VANET Routing Protocols and Implementation of a VANET Terminal. In: International Conference on Computer Technology, Electronics and Communication (ICCTEC). 2017;p. 1248-1252. Available from: https://doi.org/10.1109/ICCTEC.2017.00272.

3) Ramamoorthy R, Thangavelu M. An improved distance-based ant colony optimization routing for vehicular ad hoc networks. International Journal of Communication Systems. 2020;33(14):e4502. Available from: https://dx.doi.org/10.1002/dac.4502.

4) Gawas MA, Govekar SS. A novel selective cross layer based routing scheme using ACO method for vehicular networks. Journal of Network and Computer Applications. 2019;143:34-46. Available from: https://dx.doi.org/10.1016/j.jnca.2019.05.010.

5) Dahan F, Hindi KE, Mathkour H, AlSalman H. Dynamic Flying Ant Colony Optimization (DFACO) for Solving the Traveling Salesman Problem. Sensors. 2019;19(8):1-28. Available from: https://dx.doi.org/10.3390/s19081837.

6) Kumar P, Verma A, Singhal P. VANET Protocols with Challenges- A Review. In: 2019 6th International Conference on Computing for Sustainable Global Development (INDIACom). 2019;p. 598-602. Available from: https://ieeexplore.org.ieee.org/document/8991260.

7) Zaidi T, Faisal S. An Overview: Various Attacks in VANET. In: 4th International Conference on Computing Communication and Automation (ICCCA). 2018;p. 1-6. Available from: https://doi.org/10.1109/CCAA.2018.8777538.

8) Khoza E, Tu C, Owolawi PA. Decreasing Traffic Congestion in VANETs Using an Improved Hybrid Ant Colony Optimization Algorithm. Journal of Communications. 2020;15(9):676-686. Available from: https://dx.doi.org/10.12720/jcm.15.9.676-686.

9) S SS, S VCS. A Multi-agent Ant Colony Optimization Algorithm for Effective Vehicular Traffic Management. In: Tan Y, Shi' Y, Tuba M, editors. ICSI 2020: Advances in Swarm Intelligence . Springer. 2020;p. 640-647. Available from: https://doi.org/10.1007/978-3-030-53956-6_59.

10) Kanellopoulos D, Sharma VK. Survey on Power-Aware Optimization Solutions for MANETs. Electronics. 2020;9(7). Available from: https://dx.doi.org/ 10.3390/electronics9071129.

11) Jindal V, Bedi P. An improved hybrid ant particle optimization (IHAPO) algorithm for reducing travel time in VANETs. Applied Soft Computing. 2018;64:526-535. Available from: https://dx.doi.org/10.1016/j.asoc.2017.12.038.

12) Srivastava A, Prakash A, Tripathi R. An adaptive intersection selection mechanism using ant Colony optimization for efficient data dissemination in urban VANET. Peer-to-Peer Networking and Applications. 2020;13:1375-1393. Available from: https://doi.org/10.1007/s12083-020-00892-8.

13) Iwendi C, Uddin M, Ansere JA, Nkurunziza P, Anajemba JH, Bashir AK. On Detection of Sybil Attack in Large-Scale VANETs Using Spider-Monkey Technique. IEEE Access. 2018;6:47258-47267. Available from: https://dx.doi.org/10.1109/access.2018.2864111.

14) Hajlaoui R, Alsolami E, Moulahi T, Guyennet H. Construction of a stable vehicular ad hoc network based on hybrid genetic algorithm. Telecommunication Systems. 2019;71(3):433-445. Available from: https://dx.doi.org/10.1007/s11235-018-0513-6.

15) Mavrovouniotis M, Li C, Yang S. A survey of swarm intelligence for dynamic optimization: Algorithms and applications. Swarm and Evolutionary Computation. 2017;33:1-17. Available from: https://dx.doi.org/10.1016/j.swevo.2016.12.005.

16) Faisal S, Zaidi T. Timestamp Based Detection of Sybil Attack in VANET". International Journal of Network Security. 2020;22(3):399-410. Available from: 10.6633/IJNS.202005_22(3).05.

17) Elhoseny M. Intelligent firefly-based algorithm with Levy distribution (FF-L) for multicast routing in vehicular communications. Expert Systems with Applications. 2020;140(112889). Available from: https://dx.doi.org/10.1016/j.eswa.2019.112889.

18) Bao X, Li H, Zhao G, Chang L, Zhou J, Y L. Efficient clustering V2V routing based on PSO in VANETs. Measurement. 2020;152(107306). Available from: https://doi.org/10.1016/j.measurement.2019.107306.

19) Du B, Wei Q, Liu R. An improved quantum-behaved particle swarm optimization for endmember extraction. Transactions on Geoscience and Remote Sensing. 2019;57(8):6003-6017. Available from: https://doi.org/10.1109/TGRS.2019.2903875.

20) Shrestha R, Bajracharya R, Nam SY. Challenges of Future VANET and Cloud-Based Approaches. Wireless Communications and Mobile Computing. 2018;2018:1-15. Available from: https://dx.doi.org/10.1155/2018/5603518.

21) Tong L, Du B, Liu R, Zhang L. An Improved Multiobjective Discrete Particle Swarm Optimization for Hyperspectral Endmember Extraction. IEEE Transactions on Geoscience and Remote Sensing. 2019;57(10):7872-7882. Available from: https://dx.doi.org/10.1109/tgrs.2019.2917001.

22) Xu M, Du B, Fan Y. Endmember Extraction From Highly Mixed Data Using Linear Mixture Model Constrained Particle Swarm Optimization. IEEE Transactions on Geoscience and Remote Sensing. 2019;57(8):5502-5511. Available from: https://dx.doi.org/10.1109/tgrs.2019.2899826.

23) Bello-Salau H, Aibinu AM, Wang Z, Onumanyi AJ, Onwuka EN, Dukiya JJ. An optimized routing algorithm for vehicle ad-hoc networks. Engineering Science and Technology, an International Journal. 2019;22(3):754-766. Available from: https://dx.doi.org/10.1016/j.jestch.2019.01.016.

24) Katsaros K, Dianati M, Tafazolli R, Kernchen R. CLWPR-a novel cross-layer optimized position based routing protocol for VANETs. Vehicular Networking Conference (VNC). 2011;p. 139-146. Available from: https://dx.doi.org/10.1109/VNC.2011.6117135. 
25) Habboush AK. Ant Colony Optimization (ACO) Based MANET Routing Protocols: A Comprehensive Review. Computer and Information Science. 2019;12(1):82. Available from: https://dx.doi.org/10.5539/cis.v12n1p82.

26) Sinwar D, Sharma N, Maakar SK, Kumar S. Analysis and comparison of ant colony optimization algorithm with DSDV, AODV, and AOMDV based on shortest path in MANET. Journal of Information and Optimization Sciences. 2020;41(2):621-632. Available from: https://dx.doi.org/10.1080/02522667. 2020.1733193.

27) Sharma I, Ramkumar KR. A survey on ACO based multipath routing algorithms for ad hoc networks. International Journal of Pervasive Computing and Communications. 2017;13(4):370-385. Available from: https://dx.doi.org/10.1108/ijpcc-d-17-00015.

28) Jyothi K, Jackson JC. A time-based approach for solving the dynamic path problem in VANETS-an extension of ant Colony optimization. Journal of Engineering Science and Technology. 2004;13(3):813-821.

29) Maheshwari S, Bhardwaj M. Secure Route Selection in Manet Using Ant Colony Optimization. American Journal of Networks and Communications. 2015;4(3-1):54-56. Available from: https://dx.doi.org/10.11648/j.ajnc.s.2015040301.19.

30) Abbas F, Fan P. Clustering-based reliable low-latency routing scheme using ACO method for vehicular networks. Vehicular Communications. 2018;12:6674. Available from: https://dx.doi.org/10.1016/j.vehcom.2018.02.004.

31) Ramakrishnan B, Sreedivya S, Selvi M. Adaptive routing protocol based on cuckoo search algorithm (ARP-CS) for secured vehicular ad hoc network (VANET). Available from. 2015;2:173-178. 